\title{
Performance and Applications of Ionic Electrospray Micro-Propulsion Prototypes
}

\author{
Daniel G. Courtney* ${ }^{*}$, Simon Dandavino ${ }^{\dagger}$ and Herbert Shea ${ }^{\ddagger}$ \\ Microsystems for Space Technologies Laboratory(LMTS), Ecole Polytechnique Fédérale de Lausanne (EPFL) \\ Neuchatel, CH-2002, Switzerland
}

\begin{abstract}
Electric propulsion systems using electrospray ion sources are a scalable and high specific impulse technology which could enable small spacecraft to perform high $\Delta V$ missions. This paper presents an overview of a recently developed source design which achieves 10's of $\mu N$ of thrust at less than $1 W$ of input power with a specific impulse up to $\sim 3000 s$. Demonstration devices, fabricated conventionally without microfabrication methods yet with an active area of $\sim 1 \mathrm{~cm}^{2}$, emit positive and negative ion beams from an ionic liquid passively supplied from a coupled porous reservoir. Directly measured thrusts (up to $\sim 28 \mu N$ ) from two simultaneously operating thruster modules are shown to be consistent with summing the calculated total force from each module. The two particle beams are configured to be at opposite polarity, in progression towards a charge neutralized system without a dedicated neutralizer. Influences of reservoir pore size and filling state are discussed in the context of performance and lifetime. Specifically, recent results have demonstrated that increasing the reservoir pore size can induce significant droplet or heavy particle populations within an otherwise ionic beam. Large reductions in specific impulse and propulsive efficiency due to these transitions are discussed here. For example, the calculated specific impulse of a negative $\mathbf{E M I}_{-\mathrm{BF}}$ beam could be reduced from $\sim 2800 s$ to $\sim 700 s$ by changing the reservoir pore size alone. Meanwhile, capillary actions within the reservoir aid in containing liquid via a negative Laplace pressure, thereby preventing lifeending liquid to extractor grid bridges/shorts. Finally, the technology status is reviewed through highlighting critical developments required to arrive at a functional, and applicable, propulsion system.
\end{abstract}

\section{Introduction}

Many small satellites are launched without active propulsion or employ systems characterized by low total impulse capabilities and/or efficiency. Electrospray micropropulsion attempts to fill this technology gap by providing a high efficiency, high specific impulse system enabling a wider spectrum of mission objectives.

In electrospray propulsion systems (eg. [1-9]) structures, the emitters, are used to guide liquid from a reservoir to a region of high electric field at their peak. The field de-stabilizes the meniscus leading to charged particle emission. These particles are subsequently accelerated to form a high velocity beam. As a propulsion system this inherently small and efficient ${ }^{10}$ mechanism enables a high specific impulse $\left(I_{s p}\right)$ propulsion system well suited for small satellites. Furthermore, the use of room temperature ionic liquid (IL) propellants with near-zero vapor pressure has enabled passive feeding without any active pumps or pressurized storage tanks $\mathrm{s}^{5-7,9,11}$ a further benefit to small satellites; where physical resources are highly constrained.

When IL is supplied passively, or at a controlled but very low flow rate, sources have been shown to be capable of approaching a Purely Ionic Regime (PIR) of operation. ${ }^{11,12}$ Here the electrospray comprises only ions without any colloidal droplets and the source is sometimes referred to as an Ionic Liquid Ion Source

\footnotetext{
${ }^{*}$ Postdoctoral Researcher, EPFL-IMT-LMTS, AIAA Member

${ }^{\dagger}$ Deputy Director, EPFL-eSpace

¥Associate Professor, EPFL-IMT-LMTS

Copyright (C) 2015 by the American Institute of Aeronautics and Astronautics, Inc. All rights reserved.
} 
(ILIS). ${ }^{11}$ The low mass of the constituent ions in the beam permits an $I_{s p}$ of several $1000 s$ at $\sim 1-2 k V$ accelerating potential and the highly mono-energetic ${ }^{9,13}$ beams are emitted with high efficiency. However; if even a few percent (by current) of the beam particles are due to much heavier droplets or very large cluster ions, the resultant polydispersity dramatically reduces the effective propulsive efficiency (see e.g. Ref. [14]) and specific impulse. As a result, this mixed mode of operation should be avoided and efforts have typically focused on either pressure fed droplet emissions (see e.g. [15]) or, as in the work presented here, approaching the PIR with passively fed devices.

The thrust yield from each emission site on an ILIS emitter structure is typically only a few $10 \mathrm{~s}$ of $n N$, but from a source measuring 10s of $\mu m$ or less. The studies cited above have, at multiple institutions, therefore focused on developing compact, multiplexed arrays of sources to achieve useful thrust levels while maintaining high specific performance metrics. While the Microsystems for Space Technologies Laboratory (LMTS) has previously developed microfabricated silicon capillaries, culminating in the MicroThrust program described in the next section, a new microbrication free approach, summarized in Figure 1, has now been implemented. The approach and experimental investigations of the 100's of $\mu A$ particle beams supported by the resulting devices have been recently described in two publications, Refs. $[9,16]$. This paper summarizes key findings of those results, presents new data obtained when operating two devices simultaneously (as a pre-cursor to achieving charge neutralization) and presents discussion concerning the technology status.

\section{Thruster Design and Features}

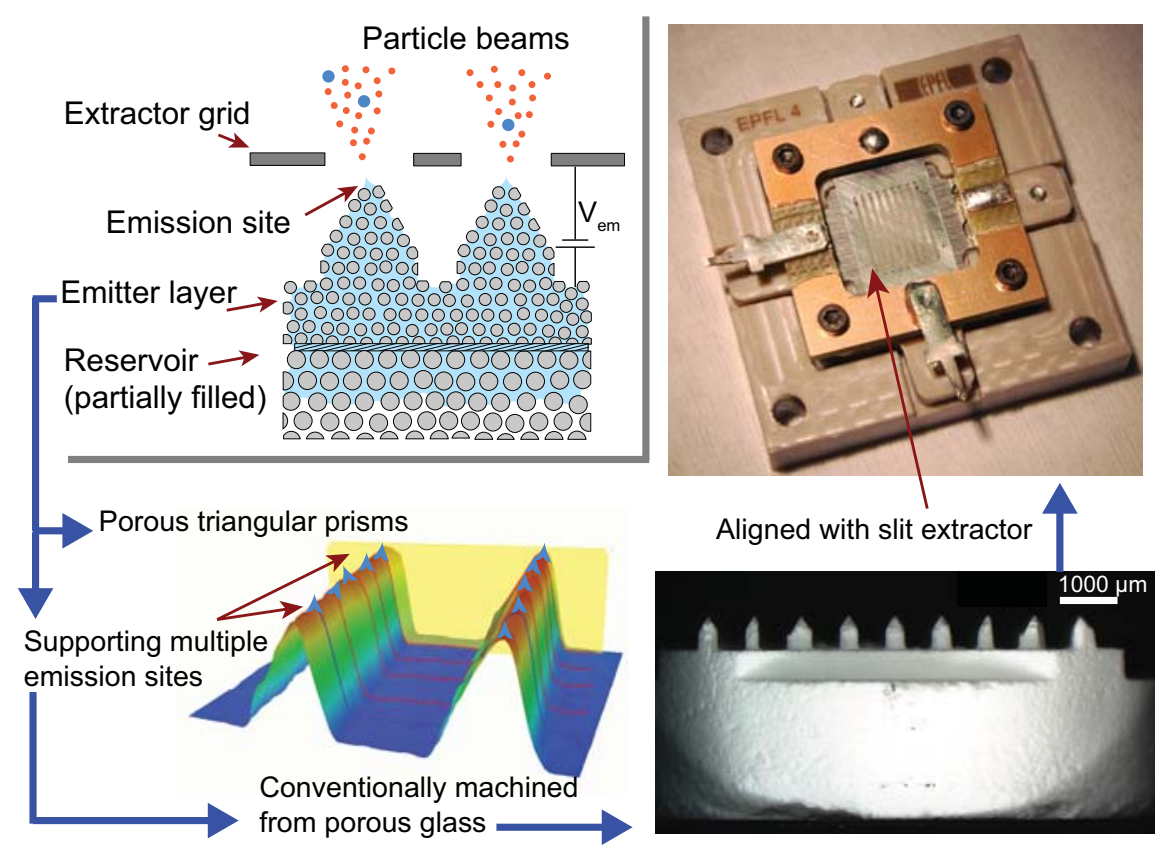

Figure 1. Triangular prisms cut from porous glass filter discs are filled with Ionic Liquid (IL) and aligned below linear extractor grids. When a strong $(\sim 2000 \mathrm{~V})$ potential is applied between the IL and extractor grid, a highly ionic particle beam is emitted. Propellant is wicked up to the emission sites from a porous reservoir layer passively, without any pumps or valves. The pore size and fill state of this reservoir layer are critical to the device operation.

\section{A. Heritage from MicroThrust Program}

Our group, within the LMTS at EPFL, previously contributed to the European "MicroThrust" project. Completed in 2013, the project was a collaboration with European academic and industry partners which sought to extend the development of microfabricated silicon capillary-type emitter arrays towards a functional breadboard system suitable for CubeSats. Operation at or near the PIR was desired to achieve high specific impulse and power efficiency; while passive feeding was sought to limit the system mass and 
complexity. Significant effort at the LMTS was devoted to creating silicon capillary emitters with inner diameters approaching $5 \mu \mathrm{m}$. This goal stemmed from previous research demonstrating that the low flow rates consistent ${ }^{12}$ with the PIR could be enforced through a large hydraulic impedance. ${ }^{17}$

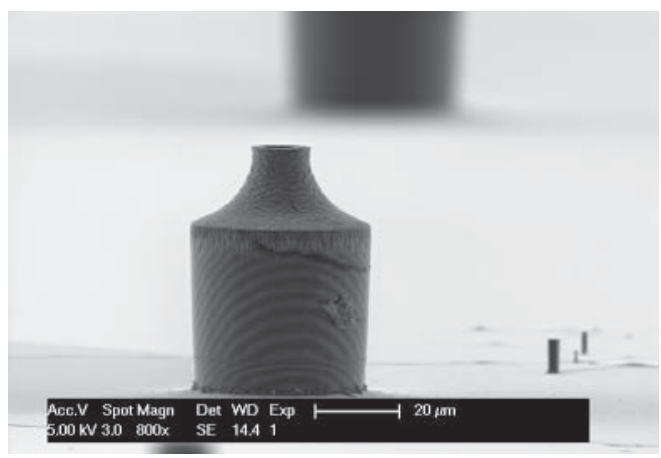

Figure 2. A silicon capillary-type microfabfricated emitter created by the LMTS during the MicroThrust program. ${ }^{8}$

While fabrication efforts approached the design target, yielding emitters with $\sim 8 \mu m$ inner diameter ${ }^{8}$ as in Figure 2, numerous operational issues arose. The devices were operated vertically, pointing downwards, with a small cup of ionic liquid pressed against each chip; effectively providing a gravity feed albeit with only a few $\mathrm{mm}$ of pressure head. Obtaining consistent wetting of each emitter was a challenge, with the meniscus frequently reaching stable points too far below the opening of the capillary to initiate a Taylor cone. Although a consistent explanation for this was not reached, the internal structure of the capillary and surface contaminations were likely contributors. When wetting was achieved, the devices often failed quickly due to IL bridging between the emitter and grid structures. Nevertheless, some devices were operated successfully yielding up to $65 \mu \mathrm{A}$ of current from an array of 121 emitters. ${ }^{18}$ However; even with the smallest capillary inner diameters and thereby highest hydraulic impedance tested, sustained operation in the PIR was elusive.

A particular success of the MicroThrust project was the implementation of an integrated dual extraction and downstream acceleration grid. The grids comprised a thin silicon extractor grid bonded to a pyrex wafer, with the latter patterned using micro-sandblasting. A metallic layer was then deposited on the top of the stack to form the accelerator electrode. See Ref. [8] for further details. Through decoupling the extraction, controlled by the emitter to extractor grid potential, and the beam energy, controlled by the acceleration to emitter potential, thrust performance and control can be improved; as discussed subsequently in this paper.

Observations of droplet content and frequent liquid-bridge failures were a particular frustration when compared with passively fed porous ${ }^{5,7}$ and externally wetted ${ }^{11}$ type emitters which have shown a high propensity for repeatable operation at or near the PIR. While the hydraulic impedance of the latter is likely high, porous emitters comprise multiple connected flow paths with pore diameters, at times, ${ }^{6}$ approaching $\sim 5 \mu \mathrm{m}$. However; compared with the MicroThrust arrangement, the integral reservoir of a bulk porous material provides a strong, effectively negative, back-pressure tending to restrain liquid from being pulled out of the substrate. We therefore desired an improved understanding of the influences of reservoir conditions, see section $\mathrm{C}$.

\section{B. Conventional Machining of High Current Density Sources}

Subsequent to MicroThrust, a new form of emitter structure, which would provide a more stable and consistent platform for development, was sought. The result is a simple method for achieving high current density ILIS (100's of $\mu \mathrm{A} / \mathrm{cm}^{2}$ ) by conventionally machining porous glass with a CNC mill. The process is described in detail within Ref. [9]. Porous borosilicate glass discs, $1 \mathrm{~cm}$ in diameter and $3 \mathrm{~mm}$ thick (Duran Group P5 grade, 1-1.6 $\mu \mathrm{m}$ pore diameters) have been cut using a mill to form triangular prisms with an apex radius of curvature of a few 10's of $\mu m$, see Figure 3 .

This geometry has been targeted to exploit two traits of porous ILIS. Firstly, despite challenges in obtaining emission consistently from silicon microcapillaries during MicroThrust, porous metal ${ }^{5,6}$ and insulating ${ }^{7}$ ILIS emitters have been successfully demonstrated from several materials and provide an integral IL path through the bulk to the emission site. Second, in Ref. [19] the propensity for porous emitters to support 


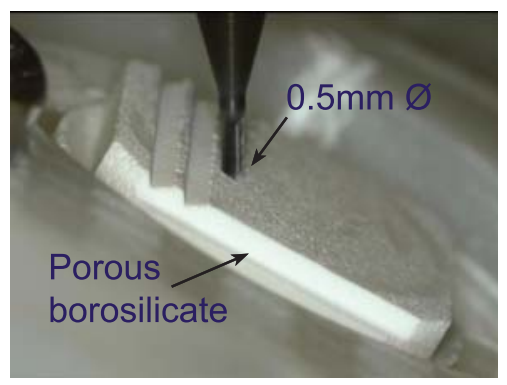

(a) Fabrication with a CNC mill.

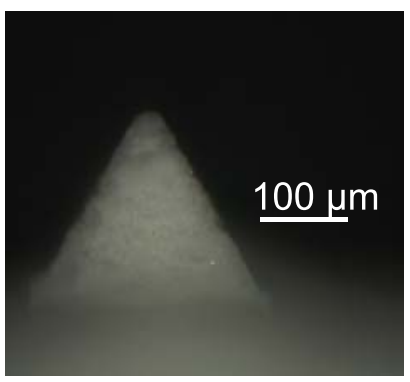

(b) Profile view of an emitter edge.

Figure 3. Triangular prisms are cut from porous borosilicate filter discs (Duran Group P5 grade) using a conventional CNC mill. Each strip is $7.5 \mathrm{~mm}$ long with an apex radius of curvature of a few 10's of $\mu \mathrm{m}$; although some regions of larger radii are typical. See Ref. [9] for details of this process.

multiple emission sites, localized approximately to pores in the vicinity of high fields, was discussed and demonstrated. Cognisant of the latter trait, high current emission necessitates a large area of porous material where the local field is sufficient to destabilize the menisci at each opening and begin emitting. The triangular prisms implemented in our approach achieve this through 9 long $(7.5 \mathrm{~mm})$ edges, with 10's of $\mu m$ radii of curvature, per filter disc. These bulk-porous structures are capable of yielding similar field enhancement to emitters in for example Ref. [6]; yet the relatively large structures present a significantly reduced challenge to fabricate.

Referring to Fig. 1, after fabrication each disc is coupled to a second porous glass disc via a layer of filter paper and mounted below a slit extractor positioned roughly inline with the emitting edges. This extractor, with 350 to $500 \mu \mathrm{m}$ wide openings, is presently fabricated from $100 \mu \mathrm{m}$ thick laser cut molybdenum sheets. Further benefits, and drawbacks, of the fabrication and assembly approaches are available in Ref. [9].

\section{Reservoir Selection and Filling: Critical Parameters}

Our investigation into the influences of the reservoir layer, coupled to an emitting layer as shown in Figures 1 and 5 , has revealed it can have a profound impact on performance when propellant is transported passively. The study, described in Ref. [16], showed that by changing the pore size of a partially filled reservoir the maximum interfacial pressure jump from inside the liquid to vacuum is altered. This pressure jump is effectively negative when using a porous reservoir in vacuum, as the liquid is drawn back into the material. When negative, the meniscus of Taylor cone is known to be curved and tends to flatten at length scale governed by the pressure ${ }^{20}$ leading to Taylor cones with base diameter on the order of the reservoir pore size. When using an emitting layer with a pore size smaller than the reservoir, the former will fill until in equilibrium with the reservoir. Hence it is the reservoir which governs the static interfacial pressure jump and thereby maximum Taylor cone size (since viscious losses would only further reduce the pressure at the apex). In the cited work, we demonstrated that reservoirs with larger pore size require lower starting voltages and, critically, that reservoir pore size can significantly alter the ability to obtain operation in the PIR; for a consistent emitter porosity. Specifically, for both the ILs EMI-Im and EMI-BF 4 droplets and cluster ions dominated the mass flow rate when using reservoirs with large pore diameter $(>100 \mu m)$. When the same devices were coupled to reservoirs with pores measuring 10's of $\mu m$, the overall mass flow rates dropped by up to 15 times and, for both liquids, the mass flow of heavy species was less than that due ions and singly or doubly solvated-ions.

The impacts of these transitions on performance metrics are demonstrated in Figure 4 where the data of Ref. [16] has been subjected to further analysis to estimate the propulsive efficiency (due to polydispersity alone) and specific impulse. Each sub-figure presents data from a single emitter structure coupled to different porous reservoirs. The 'bubble point pressure' is an IL specific quantification of the (negative) interfacial pressure jump enforced by each reservoir; smaller pores leading to higher bubble point pressure and a larger negative pressure jump. Referring to Fig. 4(b), the device emitting EMI-Im approached the PIR when using a reservoir with relatively small pores, for a bubble point approaching $9 k P a(10-16 \mu m$ pores, Duran Group $\mathrm{P} 4$ grade). The device thereby archived nearly $80 \%$ polydispersive efficiency and $\sim 2800 s$ specific impulse 


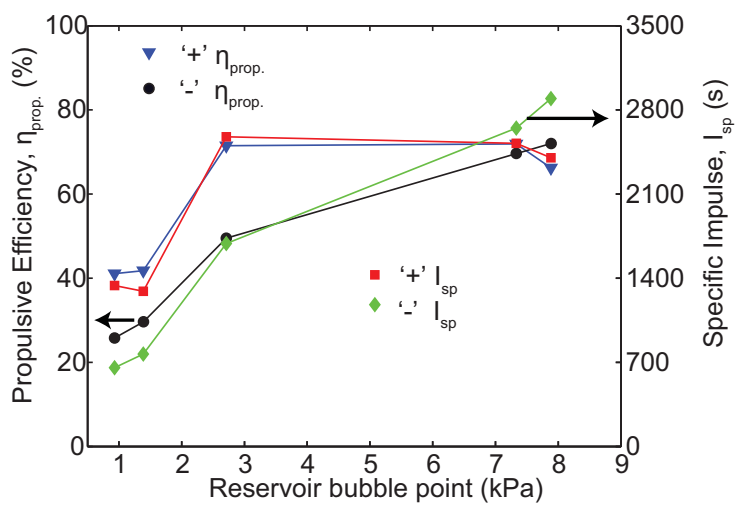

(a) $\mathrm{EMI}_{-} \mathrm{BF}_{4}$

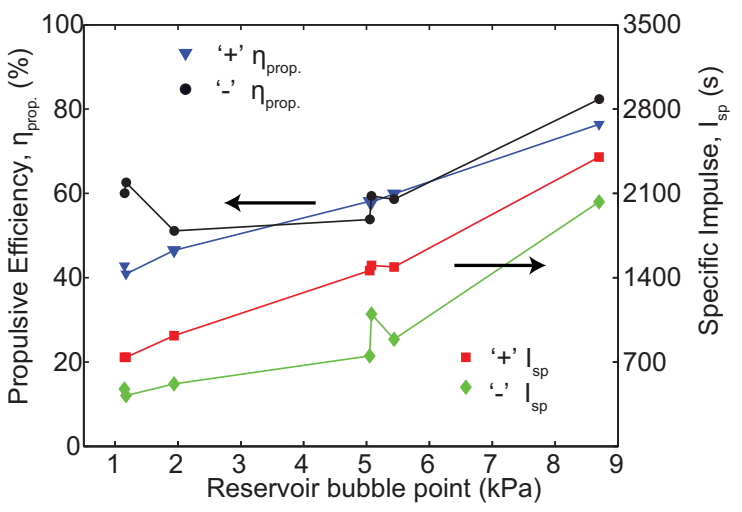

(b) EMI-Im

Figure 4. Changing the pore size of a porous reservoir alters the static maximum internal pressure of the system. For the same emitting layer, smaller pores in the reservoir, corresponding to larger bubble point, lead to an increasingly higher ion content and thereby high efficiency and specific impulse.

(at roughly $2000 \mathrm{~V}$ ). When coupled to a Duran Group P1 grade reservoir, with pores of 100 to $160 \mu \mathrm{m}$ and a bubble point of $\sim 1 k P a$, a significant droplet population was introduced without any change to the emitters. The correspondingly inefficient mixed mode operation reduced calculated efficiently to $40-60 \%$ and specific impulse to $<1000 s$; relatively dramatic changes in performance. Similar trends can be observed in Fig. 4(a) emitting EMI-BF 4 and further data, including Time of Flight traces are available in Ref. [16].

The importance of reservoir configuration is not restricted to performance. In the cited study, attempts were made to take measurements using a small rear mounted reservoir 'cup' to emulate conditions similar to those during the MicroThrust program discussed above. With the reservoir layer then completely saturated/filled it's internal pore structure no longer governed the liquid interfacial pressure; thereby providing a limiting case of (nearly) zero feeding pressure. However, as in the MicroThrust program, attempts to operate in this state were plagued by frequent shorts between the emitting structure and the extracting grid; issues which have not been observed to date when using an unsaturated reservoir. Figure 5 presents two apparent risks which may contribute to failures in this configuration, or any wherein all porous layers are saturated and therefore feed pressure is zero or positive with respect to vacuum. Firstly, depending on the wettability of the porous emitting layer and thereby the degree to which menisci at its surface may oppose a small positive pressure, liquid could be drawn through the emitting substrate and flood the surface. This would risk contact with the extractor layer, particularly after or during application of an applied field which will also tend to draw liquid through the emitter layer. In contrast, like a sponge, an unfilled porous reservoir will always tend to draw liquid back into equilibrium with the emitter layer. Secondly, as discussed above, in a passively fed condition (meaning no flow rate control is available) the size of the Taylor cone base will be governed by the interfacial pressure jump in the liquid. If too low in magnitude, this could lead to Taylor cones which are large enough to contact the extractor grid. Through governing the interfacial pressure, un-filled reservoirs ensure small Taylor cones with bases on the order of the reservoir pore size (eg. 10's of $\mu \mathrm{m}$ ) thereby reducing this failure risk. In both failure modes the near-zero vapour pressure of IL and lack of forced flow control present major challenges to recovering from a short on orbit and could thereby result in the end-of-life of the thruster module.

\section{Thruster Performance}

We have characterized devices at two facilities, the LMTS and the European Space Agency (ESA) Propulsion Laboratory (EPL) at Noordwijk, the Netherlands. At the LMTS, devices have been diagnosed using, precision current detectors, Time of Flight (ToF) spectrometry and Retarding Potential Analyzers (RPAs). At the EPL, the thrust output has been measured directly using Mettler-Toledo precision balances. In both cases devices are tested under vacuum, typically $<5 \times 10^{-5}$ mbar. Complete descriptions of both facilities are available in Ref. [9]. At all times in the data presented below, devices have been operated with partiallyfilled Duran Group P3 grade reservoirs; consistent with highly ionic (near PIR) operation when emitting 


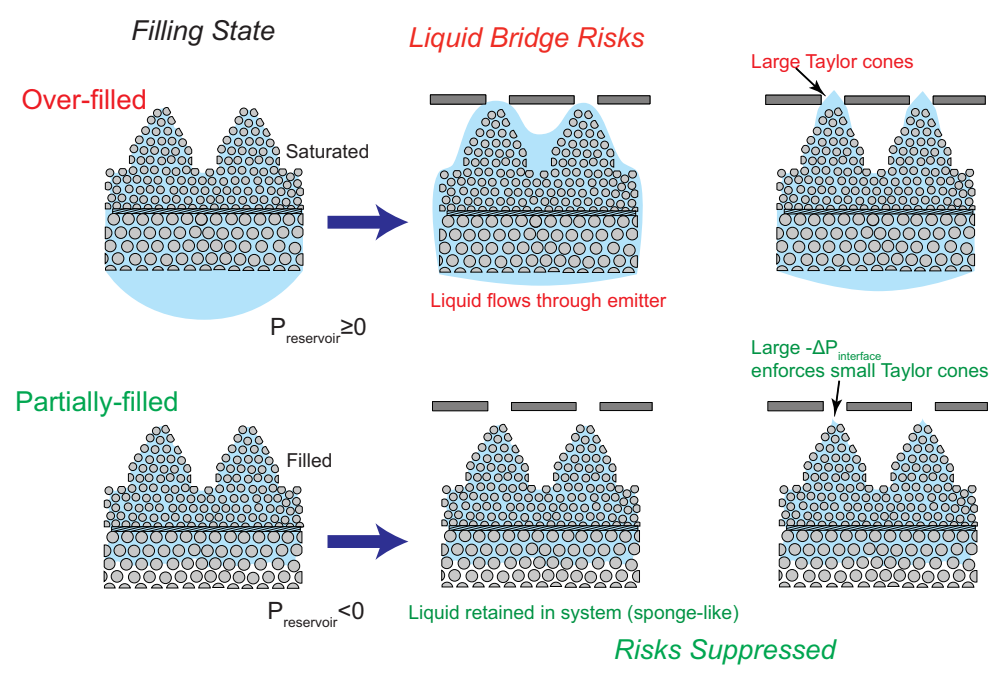

Figure 5. Reservoir conditions can contribute to life-ending failures due to liquid bridging between the emitter and extractor grid. Over-filled reservoirs may saturate the porous emitting layer and slowly wick through that layer until shorted, or lead to large Taylor cones spanning multiple pores and drawn (by the applied electric field) into contact with the extractor grid.

\section{$\mathrm{EMI}_{-\mathrm{BF}}$. $^{2}$}

\section{A. Individual Module Performance}

A detailed description of individual module performance, including a suite of diagnostic measurements and direct thrust measurements are available in Ref. [9]. Each $1 \mathrm{~cm}$ diameter porous disc, with 9 emitting strips, has been shown to yield 100's of $\mu A$ (up to $500 \mu A$ to date) of bi-polar emission with emitter voltages $<2500 \mathrm{~V}$. Figure 6(a) provides an example of the IV behaviour when emitting the IL EMI-Im during thrust acquisition. The simultaneously directly measured thrust is presented versus applied power in Figure 6(b). Outputs in the 10's of $\mu N$ were recorded from each device, see the cited material for more extensive data sets from multiple devices.

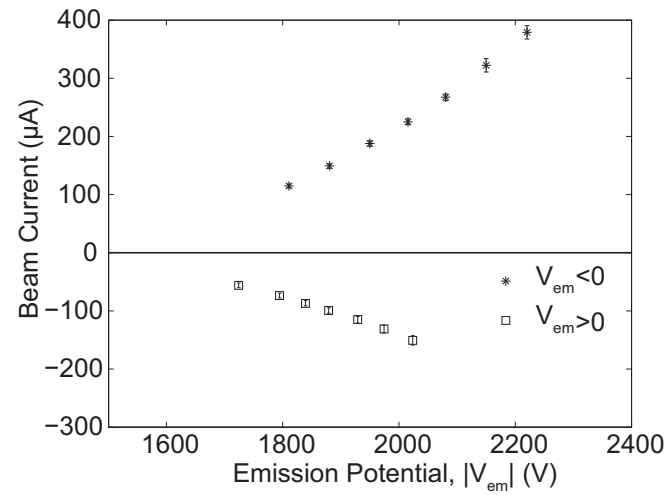

(a) Current vs. voltage while measuring thrust.

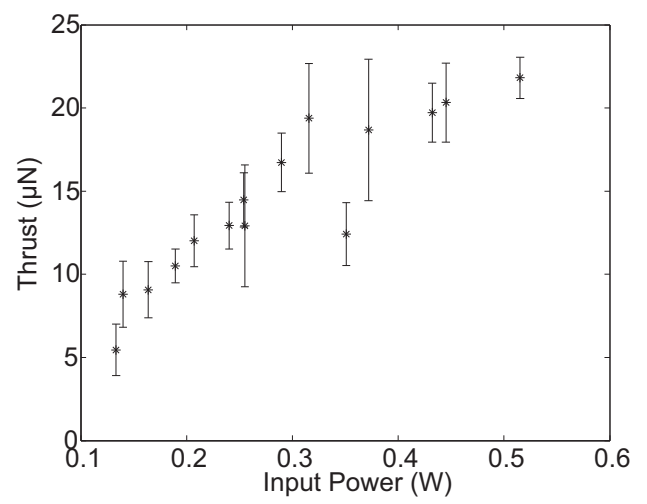

(b) Sample direct thrust measurements.

Figure 6. Direct thrust measurements have been made at the ESA Propulsion Laboratory (EPL). After the preliminary data presented here, more extensive data sets were collected and analyzed, see Ref. [9].

An analysis of the diagnostic data demonstrated that the thrust output at each polarity was typically consistent, to within a few $\mu N$, with equation 1 . Here $\alpha$ is a polarity dependent coefficient accounting for beam composition (including fragmentation ${ }^{21}$ ), energy deficits and beam collimation. During those tests, the devices were operated in a manner targeting equal thrust output a both the positive and negative polarity 
while alternating the polarity at $1 \mathrm{~Hz}$ to suppress electrochemical degradation. ${ }^{22}$ The coefficients $\alpha^{+}$and $\alpha^{-}$were nearly equal when emitting EMI-BF 4 . However, when emitting negative beams of EMI-Im, a heavy particle, or droplet, population was detected; leading to less targeted negative current than positive, as can be seen in Fig. 6(a). To compensate for differing current levels, the alternation duty cycle was adjusted such that an equal total charge was transferred per polarity over each period was maintained; as is likely necessary for effective electrochemical suppression. ${ }^{23}$

$$
T^{ \pm}=\alpha^{ \pm}\left|I_{\text {beam }}\right| \sqrt{|V e m|}
$$

The consistency between indirectly and directly measured thrusts has permitted estimates of propulsive efficiency and specific impulse capabilities. Emitting the IL EMI-BF 4 , a propulsive efficiency of roughly $65 \%$ was calculated with a specific impulse of $\sim 3200 \mathrm{~s}$ at $0.5 \mathrm{~W}$ of input power (with $20 \mu \mathrm{N}$ thrust output). Polydispersity in the beam was the most significant inefficiency; however energy deficits up to $\sim 7 \%$, beam divergence (an effective $\sim 20^{\circ}$ beam angle) and grid interception (few \%) all contributed. Physical inspections showed that the latter may have been largely due to beams emanating from the abrupt ends of each emitter edge and directed into the grid; this loss may therefore be suppressed through design improvements. When emitting EMI-Im, large anions and a small droplet propulsion led to higher thrusts, up to $50 \mu N$ at $<1 \mathrm{~W}$, at the expense of specific performance, due to both the relatively large mean particle mass and the accompanying polydispersity in the beam; with a calculated $\sim 1500 s$ specific impulse and $\sim 50$ $\%$ propulsive efficiency at $0.5 \mathrm{~W}$.

\section{B. Dual Module Operation}

The ability to emit positive and negative ion beams could enable operation without a dedicated neutralizer. Instead two modules could be operated simultaneously, one emitting positive particles and the other negative with identical current magnitudes. To explore and demonstrate this concept, we have recently completed a test campaign at the EPL using new devices as in Figure 7. These demonstration thrusters include two emitting modules housed within a $27 \mathrm{~mm} \times 57 \mathrm{~mm} \times 6 \mathrm{~mm}$ PEEK mount. Each module includes an emitting disc (with 9 strips) and an isolated reservoir, coupled in the same manner as in Refs. [9] and [16].

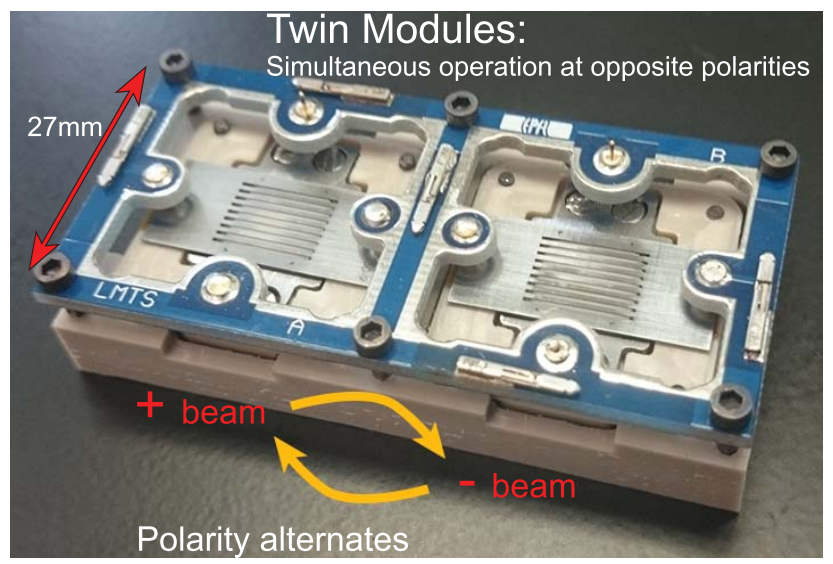

Figure 7. Recent iterations of the device include two modules in a single mount which are operated simultaneously at opposite polarity to permit charge neutralization.

Figure 8 presents sample data from this experiment. Each module (labelled 'A' and 'B') emits a beam of either positive or negative particles (using the IL EMI-BF 4 here), Figure 8(a). The propellant is conductive, hence IL in each reservoir is polarized to the same potential as the emitter layer coupled to it. The reservoirs are therefore electrically isolated and risks of electrochemical degradation are local to each, specifically at its interface between the IL and a polarizing electrode. Correspondingly, as shown in the Figure, the beam polarity must again be alternated back and forth between each module; at $1 \mathrm{~Hz}$ here.

In Figure 8(b) thrust output at the current levels indicated in Fig. 8(a) has been calculated using (without customization) the coefficients listed in Ref. [9] (and comprising $\alpha$ in Eqn. 1) to these emitter arrays. The thrust has been measured directly using a similar arrangement as in the reference yet using 
a Mettler Toledo AX504 model balance instead of the lower resolution XP2004-S model used previously. As shown, the calculated thrusts from each module sum to a level in good agreement with that measured directly. While this example demonstrates a peak thrust of roughly $28 \mu N$, a force roughly twice as large would be obtained if each module was operated at the peak currents measured from individual modules to date (up to $300 \mu \mathrm{A}$ per module emitting EMI-BF 4 ).

The consistency between the directly measured thrust data and calculated thrusts is encouraging considering the calculations were made using previously determined diagnostics for a different EMI-BF 4 module. Given this agreement, specific performance levels ( $\sim 65 \%$ performance efficiency and $\left.\sim 3000 s I_{s p}\right)$ are anticipated to have been maintained. Furthermore, this result reflects the consistent nature of the fabrication and assembly scheme employed; further reinforcing the suitability of this approach for continued development towards a complete breadboard thruster. Finally, the result is a first step towards demonstrating the scalability of these sources.

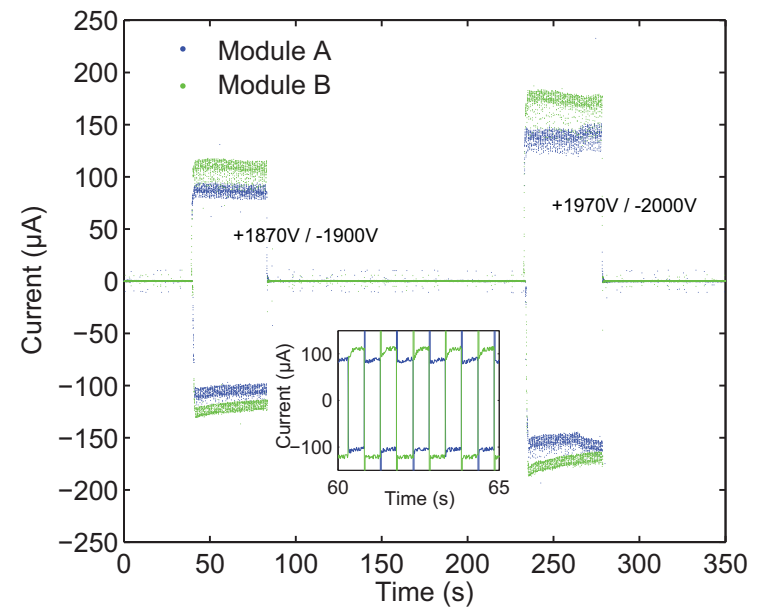

(a) Measured currents.

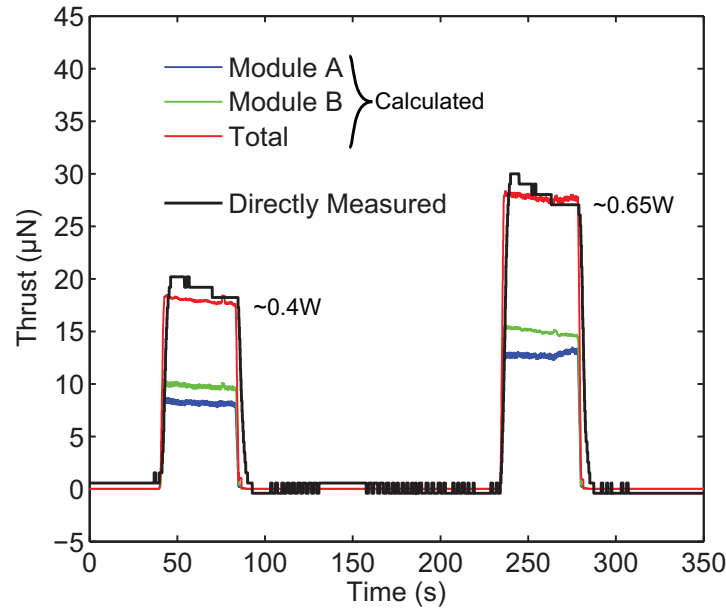

(b) Calculated and directly measured thrusts.

Figure 8. Demonstration of operating a device as in Figure 7 with the two modules configured to emit EMI$\mathrm{BF}_{4}$ beams of opposite polarity. Calculated thrust levels, using the coefficients presented in Ref. [9], for both modules sum to a level in good agreement with the thrust directly measured using a balance.

While the presented data confirms dual mode operation, charge neutralization would not be achieved implicitly by this arrangement; given the currents were not well matched in Figure 8(a). Treating a small satellite as a $20 \mathrm{~cm}$ radius conductive sphere, its capacitance would be only $\sim 20 \mathrm{pF}$. A $10 \mu \mathrm{A}$ current imbalance would charge the spacecraft to $200 \mathrm{kV}$ with respect to its environment in a $0.5 s$ half-period, or reach $2 \mathrm{kV}$ in less than $5 \mathrm{~ms}$. Active controls are therefore being tested which ensure any inequalities in beam current are nullified at 100's of $\mathrm{Hz}$ to $\mathrm{kHz}$ rates. These tests, performed while measuring the voltage of the electrically floating thruster and power processing systems with respect to ground, are ongoing at the time of writing.

\section{Mission Applicability and Technology Status}

The present prototypes have been designed as demonstrations only, targeting performance validation and an understanding of reservoir conditions.

Compared with, for example, the specifications targeted by electrospray systems supported by NASA's Game Changing Development Program, ${ }^{24}$ the specific performance abilities presently achieved are encouraging. That program called for an $I_{s p}$ greater than $1500 \mathrm{~s}$, thrusts in excess of $100 \mu \mathrm{N}$ at less than $10 \mathrm{~W}$ input power and with a system efficiently in excess of $70 \%$. Considering, for example, Fig. 8(b) where nearly $30 \mu N$ of thrust was directly measured at approximately $0.65 \mathrm{~W}$ input power and with a high (calculated) specific impulse approaching $3000 \mathrm{~s}$, a total thrust of $100 \mu N$ appears well within reach at less than 10 $W$. However, while propulsive efficiencies close to $70 \%$ have been calculated at the module level, the total efficiency would likely reduce significantly as a complete system including a PPU.

To date, devices have been operated with small $1 \mathrm{~cm}$ diameter, $3 \mathrm{~mm}$ tall reservoir layers which, as 
described above, are intentionally never completely filled. Their useful propellant, prior to depletion of the dedicated reservoir, has typically been only $\sim 50 \mu L$ per module (with the $\sim 70-90 \mu L$ within emitter layer itself treated here as 'unusable' due to the expected change in operating voltage). At a $3000 s I_{s p}$ and using EMI-BF ${ }_{4}$, this corresponds to a small total impulse capability of $\sim 1.8 \mathrm{Ns}$, or a run time of only 25 hours at $20 \mu N$ per module. To date devices have been operated for a maximum of roughly 6.5 hours.

While these low impulse capabilities may be of use to some specific CubeSat missions, for example demonstrating precision attitude control or formation flight, it is evident that for systems capable of 10's or 100 's of $\mathrm{m} / \mathrm{s}$ of $\Delta V$ a larger propellant capacity is required as the device design develops. Those developments should be accompanied by increasingly applicable tests of lifetime limitations. Given our findings considering the importance of reservoir pore size, the implementation of such an expansion must include considerations of the high dry mass per volume of stored IL associated with using entirely porous reservoirs.

Although at this stage liquid bridging has not been observed, a 4.5 hour continuous operation test ${ }^{9}$ did reveal a gradual degradation in performance before the test was ended voluntarily. There, a targeted thrust level of $20 \mu N$ was enforced using a closed loop controller while recording the emitter voltage required. This voltage increased from $+2100 /-2030 \mathrm{~V}$ to $+2450 /-2500 \mathrm{~V}$ over the course of the test. Subsequent inspection revealed significant grid erosion was incurred near the end of each emitter strip and the surface was discoloured. To a degree, sputtered material may have contributed to a reduction in current at a given voltage. Although the emission potential was alternated at $1 \mathrm{~Hz}$ during that test, the electrical contact to the IL was made with aluminum (which is not common to other ILIS tests in the literature) and the degree to which electrochemical effects were suppressed requires further investigation.

At the device level, an improved understanding of the emission site distribution along each edge is required to both optimize the geometry and improve ion optics. The latter is of particular relevance in working towards an efficient multi-grid implementation, like that implemented in the MicroThrust program. Through decoupling current and total energy control, such a configuration could be vital to controlling output (thrust/specific impulse) in addition to charge and electrochemical balances.

As an example of the control subtleties required: when employing a dual (or even several pair) operating configuration, careful attention must be made to achieve both charge neutralization of the complete thruster and a charge transfer balance at each reservoir. Consider a pair of modules where the 'A' module is emitting $+100 \mu \mathrm{A}$ and $-80 \mu \mathrm{A}$. As described, active compensation could be used to ensure the 'B' module emits $100 \mu A$ and $+80 \mu A$ and overall charge neutrality is obtained. However; over each period of alternation the 'A' reservoir would have transferred a net of $+20 \mu \mathrm{A}$ and the 'B' reservoir $-20 \mu \mathrm{A}$. While globally balanced, the isolated IL reservoirs in such a configuration would apparently accrue an excess of charges, increasing the risk of electrochemical breakdowns. Instead, a suitable controller should attempt to null this period averaged current while also balancing net charge output. This could be effected with an inner extraction grid control while the outer grid could permit simultaneous thrust/specific impulse control at high precision.

\section{Conclusion}

Electrospray remains a promising technology to fill the expanding needs for propulsion systems on small satellites. Thruster prototypes developed at the EPFL Microsystems for Space Technologies Laboratory have been shown to obtain high propulsive efficiency while yielding 10's of $\mu N$ of thrust and with a specific impulse in the vicinity of $3000 \mathrm{~s}$. The IL propellant is supplied to the thrusters passively, through capillary actions and the devices are, relatively, straight forward to fabricate using conventional machining. Two thruster modules have been operated simultaneously at opposite polarity. The directly measured thrust was consistent with summing the calculated thrust from each module; a favourable result in progressing towards a scalable technology with integral charge neutralization. The importance of reservoir selection and fill state have been reviewed in the context of avoiding liquid-bridge (/short) failures and ensuring performance within the desired ion/droplet content state desired. Specifically, un-filled reservoirs with small pore size permit operation close to a purely ionic regime and suppress failures by liquid-grid shorts.

Numerous developments are required to extend these demonstration devices towards a breadboard thruster level. These includes increasing the propellant capacity, investigating and addressing lifetime limitations and significant advances in power processing and control. If implemented, the presented form of electrospray thruster would provide a simple and scalable electric propulsion option to small satellite mission designers. 


\section{Acknowledgments}

This work has been supported in part through ESA NPI contract \#4000109063/13/NL/PA. The authors would like to thank Alexandra Bulit, Davina Di Cara, Kathe Dannenmayer and Eduard Bosch Borras of the ESA Propulsion Laboratory for their assistance with the direct thrust measurements.

\section{References}

${ }^{1}$ Ziemer, J. K., Randolph, T. M., Franklin, G. W., Hruby, V., Spence, D., Demmons, N., Roy, T., Ehrbar, E., Zwahlen, J., Martin, R., and Connolly, W., "Colloid micro-newton thrusters for the space technology 7 mission," IEEE Aerospace Conference 2010, Big Sky, MT, March 2010, also AERO-2010-5446760.

${ }^{2}$ Gassend, B., Velasquez-Garcia, L. G., M., A. I. A., and Martinez-Sanchez, "A microfabricated planar electrospray array ionic liquid ion source with integrated extractor," Microelectromechanical Systems, Journal of, Vol. 18, No. 3, 2009 , pp. 679-694.

${ }^{3}$ Lenguito, G. and Gomez, A., "Development of a multiplexed electrospray micro-thruster with post-acceleration and beam containment," Journal of Applied Physics, Vol. 114, 2013, pp. 154901.

${ }^{4}$ Hill, F. A., Heubel, E. V., de Leon, P. P., and Velásquez-García, L. F., "High-throughput ionic liquid ion sources using arrays of microfabricated electrospray emitters with integrated extractor grid and carbon nanotube flow control structures," Microelectromechanical Systems, Journal of, Vol. 23, No. 5, 2014, pp. 1237-1248.

${ }^{5}$ Legge, R. S. and Lozano, P. C., "Electrospray propulsion based on emitters microfabricated in porous metals," Journal of Propulsion and Power, Vol. 27, No. 2, 2011, pp. 485-495.

${ }^{6}$ Courtney, D. G., Li, H. Q., and Lozano, P., "Emission Measurements from Planar Arrays of Porous Ionic Liquid Ion Sources," IOP Journal of Physics D, Vol. 45, No. 48, 2012, pp. 485203.

${ }^{7}$ Coffman, C., Perna, L., Li, H., and Lozano, P. C., "On the Manufacturing and Emission Characteristics of a Novel Borosilicate Electrospray Source," 49 ${ }^{\text {th }}$ AIAA Joint Propulsion Conference and Exhibit, San Jose, CA, July 2013.

${ }^{8}$ Dandavino, S., Ataman, C., Ryan, C. N., Chakraborty, S., Courtney, D. G., Stark, J. P. W., and Shea, H., "Microfabricated electrospray emitter arrays with integrated extractor and accelerator electrodes for the propulsion of small spacecraft," Jounal of Micromechanics and Microengineering, Vol. 24, No. 7, 2014, pp. 075011.

${ }^{9}$ Courtney, D. G., Dandavino, S., and Shea, H., "Comparing Direct and Indirect Thrust Measurements from Passively Fed and Highly Ionic Electrospray Thrusters," Journal of Propulsion and Power, 2015, In-press.

${ }^{10}$ Lozano, P. and Martínez-Sánchez, M., "Efficiency Estimation of EMI-BF 4 Ionic Liquid Electrospray Thrusters," $41^{s t}$ Joint Propulsion Conference Es Exhibit, Tuscon, Arizona, 2005.

${ }^{11}$ Lozano, P. and Martínez-Sánchez, M., "Ionic liquid ion sources: characterization of externally wetted emitters," Journal of Colloid and Interface Sciences, Vol. 282, No. 2, 2005, pp. 415.

${ }^{12}$ Romero-Sanz, I., Bocanegra, R., Gamero-Castano, M., de la Mora, J. F., and Lozano, P., "Source of heavy molecular ions based on Taylor cones of ionic liquids operating in the pure ion evaporation regime," Journal of Applied Physics, Vol. 94, No. 5, 2003, pp. 3599-3605.

${ }^{13}$ Lozano, P., "Energy Properties of an EMI-Im ionic liquid ion source," Journal of Physics D: Applied Physics, Vol. 39, 2006, pp. 126-134.

${ }^{14}$ Lozano, P. and Martínez-Sánchez, M., "Studies on the Ion-Droplet Mixed Regime in Colloid Thrusters," Ph.D Thesis, Massachusetts Institute of Technology, Cambridge, MA, 2003.

${ }^{15}$ Alonso-Matilla, R., Fernández-García, J., Congdon, H., and de la Mora, J. F., "Search for liquids electrospraying the smallest possible nanodrops in vacuo," Journal of Applied Physics, Vol. 116, 2014, pp. 224504.

${ }^{16}$ Courtney, D. G. and Shea, H., "Influences of Porous Reservoir Laplace Pressure on Emissions from Passively Fed Ionic Liquid Electrospray Sources," Applied Physics Letters, 2015, Submitted.

${ }^{17}$ Krpoun, R., Smith, K. L., Stark, J. P. W., and Shea, H., "Tailoring the hydraulic impedance of out-of-plane micromachined electrospray sources with integrated electrodes," Applied Physics Letters, Vol. 94, No. 16, 2009, pp. 163502.

${ }^{18}$ Ryan, C., Daykin-Iliopoulos, A., Stark, J. P. W., Salaverri, A., Vargas, E., Rangsten, P., Dandavino, S., Ataman, C., Chakraborty, S., Courtney, D., and Shea, H., "Experimental progress towards the MicroThrust MEMS electrospray electric propulsion system," 33 $3^{\text {rd }}$ International Electric Propulsion Conference, Washington, DC, October 2013, also IEPC-2013-146.

${ }^{19}$ Courtney, D. G., "Ionic Liquid Ion Source Emitter Arrays Fabricated on Bulk Porous Substrates for Spacecraft Propulsion," Ph.D Thesis, Massachusetts Institute of Technology, Cambridge, MA, 2011.

${ }^{20}$ de la Mora, J. F., "The Fluid Dynamics of Taylor Cones," The Annual Review of Fluid Mechanics, Vol. 39, 2007, pp. 217-243.

${ }^{21}$ Courtney, D. G. and Shea, H., "Fragmentation in Time-of-Flight Spectrometry-Based Calculations of Ionic Electrospray Thruster Performance," Journal of Propulsion and Power, 2015, pp. 1-5.

${ }^{22}$ Lozano, P. and Martínez-Sánchez, M., "Ionic liquid ion sources: suppression of electrochemical reactions using voltage alternation," Journal of Colloid and Interface Sciences, Vol. 280, 2004, pp. 149-154.

${ }^{23}$ Castro, S. and de la Mora, J. F., "Effect of tip curvature on ionic emission from Taylor cones of ionic liquids from externally wetted tungsten tips," Journal of Applied Physics, Vol. 105, 2009, pp. 034903.

${ }^{24}$ NASA, "Micro-Electrospray Thrusters by NASAs Space Technology Mission Directorate," Availble at http://gcd.larc.nasa.gov/wp-content/uploads/2014/01/FS-MEP_factsheet_130124.pdf/. 